\title{
An Adaptive Data Replication Technique with Online Advertising for P2P VoD Services
}

\author{
Chien-Peng Ho, Senior Member, IACSIT, and Chia-Ling Hsieh
}

\begin{abstract}
This study aims to construct an agent-driven advertising framework for allowing agent-peers to distribute ad-videos to their potential customers and robust online marketing communication channels for smooth functioning of the Video-on-Demand (VoD) systems in unstructured Peer-to-Peer (P2P) overlay networks. In particular, the framework does not rely on a centralized collection server, but depends more on agent-peers that better meet customer needs and can transform a server load into scalable bandwidths with availability and high system reliability. However, in large-scale networks, it is important to reduce the load imposed on the server resources. This paper examines the problem of characterizing and assessing the local impact of the load imposed on the peer-level underlay networks by analyzing the kurtosis properties of packet inter-arrival time distribution on a P2P network. In addition, we analyzed the observed global impact of the load imposed for film-data replication to eliminate potential network bottlenecks using an extended density-based clustering (DENCLUE) algorithm of P2P overlay traffic on the agent-level underlay. The results indicate appropriate replication effective in system performance. It also points to the enormous potential of agent-level advertising in enhancing predetermined marketing objectives.
\end{abstract}

Index Terms-Overlay Networks, Peer-to-Peer Systems, Video-on-Demand, Multimedia Communications, Internet Advertising.

\section{INTRODUCTION}

With ever-increasing attention to the study of online peer-to-peer (P2P) advertising mechanisms [1], a concomitant approach has arisen in delivering marketing messages to attract customers in order to achieve business model innovation. Existing P2P advertising systems, such as ZapShares [2], MediaDefender [3], P2Pads [4], P2Pwords [5], or PeerMatrix advertising delivery services are accomplished through combination of P2P file sharing and advertising service. Peers can issue search operations to a web portal server (i.e. Mininova [6]), which provides videos or files catalog using a keyword or comprehensive query. Thereby, the search results with ad files or videos of the advertisers might be downloaded from other peers or seeds

Manuscript received April 30, 2012; revised June 3, 2012. This work was supported in part by the "Aim for the Top University Plan" of National Taiwan Normal University and the Ministry of Education, Taiwan, R.O.C. and in part by the National Science Council under Grant 100-2631-S-003-008.

Chien-Peng Ho is with the Industrial Technology Research Institute of Taiwan, (e-mail: cpho@ itri.org.tw).

Chia-Ling Hsieh is with the Graduate Institute of Teaching Chinese as a Second Language, National Taiwan Normal University, Taiwan. (e-mail: clhsieh@ntnu.edu.tw). and viewed by the target consumers of the advertisement in $\mathrm{P} 2 \mathrm{P}$ file-sharing networks. From the point of view of advertisers, advertising and circulation revenue can efficiently track click-count totals and historical data. Compared to non-P2P, online advertising includes contextual ads on search engine results, web banner advertising, social network advertising (e.g. facebook, orkut, myspace, bebo, friendster etc.), and e-mail advertising. P2P networks are more socially-aware [7] and service-oriented [8] because they can self-organize and decentralize communication. Therefore, advertising mechanisms with P2P technology can be more productive in helping advertisers reach their target audiences through a wide range of media, which includes $\mathrm{P} 2 \mathrm{P}$ video-on-demand (VoD) service, advertising-supported (ad-funded) VoD, P2P TV, P2P file sharing networks, and other $\mathrm{P} 2 \mathrm{P}$ related technologies.

In recent research, P2P VoD service has increasingly received attention. Several on demand $\mathrm{P} 2 \mathrm{P}$ video deployments have been proposed through extensive distribution and functionalities of the VoD services analysis [9]-[11]. Moreover, there is also a multitude of advertisements payment models (i.e., cost-per-action, cost-per-click, cost-per-impression, cost-per-download, and cost-per-visitor) in video-on-demand or advertising-supported VoD channels that are being deployed [12]. In addition, internet video ads units can be placed before, during and/or after demand video content in the P2P VoD service. Two major categories of internet video ad units were in-page ad units and in-stream ad units [13]. In-page ad units are video ads embedded in a search-engine's results page, which contains search results and retrieval of advertisement tracking. In-stream ad units can be within streaming video content or played on the advertisement window. In most cases, the length of internet video ads is between 17 seconds and 4 minutes. For in-page ad units, the most common play length is 30 seconds and 15 seconds [13]. However, 40-second internet video ads were shown to be the most effective in attracting target audiences on the network [14].

This paper aims to present an agent-driven advertising framework that allows peers to distribute ad-videos to a group of customers and provide a robust online marketing communication channel [15] for smooth functioning of the VoD systems in unstructured peer-to-peer networks. In addition, providing a robust online marketing communication channel is an important factor that contributes success in both advertising effectiveness and VoD service satisfaction. Audiences are unsatisfied with some of the existing $\mathrm{P} 2 \mathrm{P} \mathrm{VoD}$ services due to poor video quality and slow video download. Advertising-supported 
video on demand might offer the best programs and services possible. Thus, the proposed system will not be done by following the issuing search operations to a web portal server, as commonly done in previous research, but by referring to characteristics of the individual peers, such as historical network data collected by peers and ad-videos promoted by agent-peers. In particular, the choice of advertising time and related videos also depends on the discretion of the advertiser in terms of increasing the visibility of the ads. In sum, the main contributions of the proposed system are as follows:

1) Ads distributing relies on $\mathrm{P} 2 \mathrm{P}$ advertising agents and not on a centralized collection point (i.e. a web portal server). This implies that the scalability and flexibility of the P2P ads service platform can be improved.

2) Certain advertising plans can be created to regulate advertisement publishing rules and the regulations can be followed by advertisement providers.

3) The network characteristics of the individual peers are integrated with the platform as a replication process to ensure smooth playback in VoD systems. High video quality and smooth video downloading can make the online marketing communication channel more robust.

The remainder of the paper is organized into four sections. Section 2 provides a brief review of the most important related work. Section 3 describes an agent-based advertising's proposed framework in VoD Service. Section 4 shows the primary performance simulation results and compares the proposed system with a web portal server-based advertising system. Finally, concluding remarks and future research directions are presented in Section 5.

\section{Related Studies On THE P2P AdVERTising SERVICE}

Peer-to-peer (P2P) advertising service should be able to target promotional messages to their preferred audience by associating the ads with a set of content and network characteristics. Three of these issues are relevant to the present discussion: the high visibility of advertisement with low delivery time, the flexibility of ad time and placement, and the corresponding scalability of ad serving works.

In recent research of [[16], [17], the use of mobile communication devices for $\mathrm{P} 2 \mathrm{P}$ advertising purposes is addressed. Zaiben Chen et al. [16] employed a solution for disseminating instant advertisements to the target audience within the area of interest in a mobile P2P network. In order to spread an advertisement message, a propagation model is used to trade off timely. On the other hand, location-based ad-distribution works by considering important physical constraints of networks such as high delivery rate, low delivery time, and flooding of messages. However, the scalability of the propagation space is limited by a specified advertising area. The work in [18] describes a P2P distributed advertising method based on a reputation approach and on the incentive-based model to overcome the problem of free riding [19]. This method organizes the service providers into a semantic overlay network and motivates peers to accept advertisements as well as lookup services in a highly scalability and dynamic manner. This reputation approach is designed to cope with the free riding problem to improve the scalability and efficiency properties.
Video-on-Demand (VoD) services have been proved to be effective in dealing with the critical issues of advertising mechanisms. Examples of dynamic ad insertion systems in VoD include AdPulse [20] and BlackArrow [21]. Furthermore, P2P network allows data sharing by a large-scale community at a lower cost than the system with centralized distribution architecture. Hence, if commercial advertising delivered through $\mathrm{P} 2 \mathrm{P}$ VoD advertising platforms are well designed, they will presumably be considered a valuable application that contributes to delivering targeted messages to the right online audience. The P2P VoD advertising service expanded the horizons of advertising, including easier segment for the audience by using video catalog, tighter integration between the video-content and ads, and more scalable ways to increase the visibility of the advertisement. From a technology point-of-view, P2P sharing can be classified into two major types: file-based and media streaming-based sharing. Media streaming-based sharing scheme is more flexible for $\mathrm{P} 2 \mathrm{P}$ VoD service and many new P2P VoD technologies are envisioned and implemented [22]. By constructing a P2P VoD system, there are three key issues in the video transmission over packet loss networks. The first is media data transmission and rendering. Typically, timely and continuously streaming delivery is required to meet the playout deadline at the receiver site. The second issue is the perceptual degradation of video quality within certain limits is often negligible. The final issue is what we call "flash crowd"- the sudden or prolonged increase in peer arrivals to the P2P system.

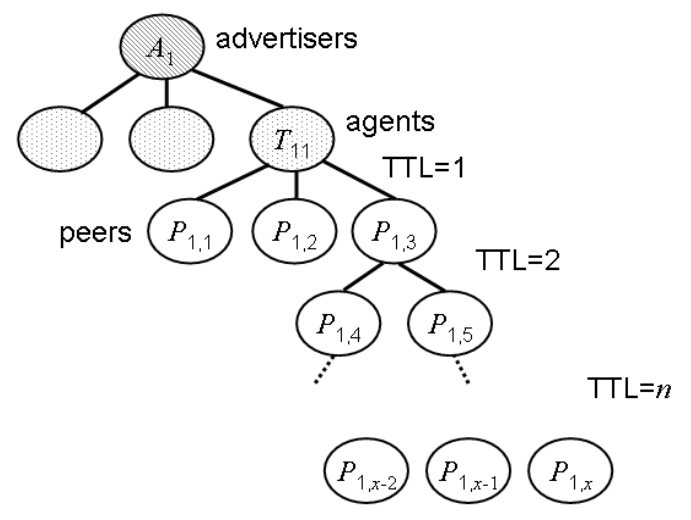

Fig. 1. Architecture of an agent-based P2P VoD advertising service

The above types of $\mathrm{P} 2 \mathrm{P}$ advertising service show that the requirement of advertising in $\mathrm{P} 2 \mathrm{P}$ streaming media system should be designed by taking the characteristics of $\mathrm{P} 2 \mathrm{P}$ communication and the behavior of $\mathrm{P} 2 \mathrm{P}$ peers. In addition, an advertising model must be adapted to the objectives of advertisers to support marketing strategies. The study thus aims to construct an advertising framework to demonstrate an ad-videos distributing principle to potential customers. One important feature of this newly born advertising platform is that it makes it possible for smooth functioning of the P2P VoD systems by including the network characteristics of peers in an online marketing communication channel. In addition, without web-based search engines such as keywords search, with some criteria being used to compose ads, the framework does not rely on a centralized collection point server (scalability and flexibility are limited by the 
server), thus, depending more on customer needs to ensure complete and successful delivery advertising service to their target audiences.

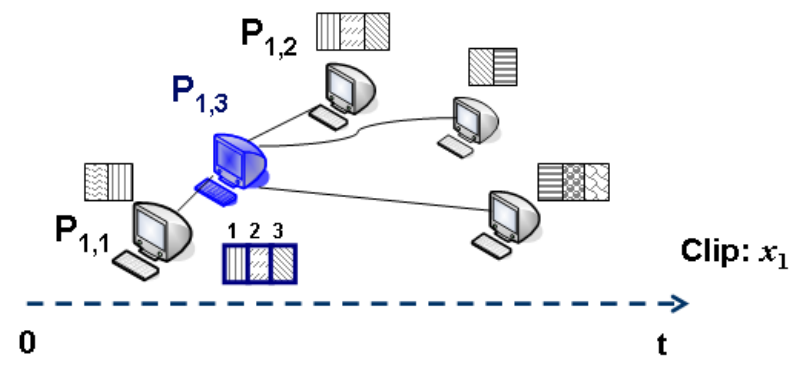

Fig. 2. An example of the blocks is temporarily stored in the video packet buffer of each peer.

\section{SYSTEM OVERVIEW}

As shown in Fig. 1, the architecture of an agent-based P2P VoD advertising service is divided into three main parts. Firstly, the overlay network is a tree rooted at the advertiser server that allows ads, selected by advertisers, to be associated with a set of particular films. For example, there is an advertiser server $A_{1}$ in the overlay network in Fig. 1. The advertiser server $A_{1}$ has a set of video ads $\left\{a_{11}, \ldots, a_{1 u}\right\}$ which are bound in a related films set (e.g. $a_{11}$ might be an ad for running shoes, the ad could associate categories of interest $\left\{f_{11}, \ldots, f_{1 v}\right\}$ which related to sport videos) selected by the advertiser. Secondly, advertising agents of $A_{1}$ are $\left\{T_{11}, \ldots, T_{1 w}\right\}$. They are trying to raise advertising effectiveness by helping advertisers convey their advertising message more closely to the intended audiences. Thus, the advertising agents are responsible for periodically collecting information among sub-tree peers in order to obtain additional attributes of audiences. Then, advertising agents can support smooth video playing in audience-peers and promote the most relevant ads to audience-peers of the sub-tree by using the attributes of $\mathrm{P} 2 \mathrm{P}$ peer. Finally, the set of audience-peers is $\left\{P_{1,1}, \ldots, P_{1, x}\right\}$, while time to live (TTL) can be treated as a search area in flooding-based unstructured P2P overlay networks. According to the network conditions and the peer characteristics in the tree, the advertiser server and the advertising agents can dramatically increase the probability of the ad files being downloaded and viewed to maximize the overall revenue generated per minute of ad videos.

\section{A. Network Awareness in Audience-Peers}

Considering the characteristics of audience-peers in $\mathrm{P} 2 \mathrm{P}$ advertising Video-on-Demand (VoD) systems, there are several attributes on the audiences side that can be used to control the runtime behavior of advertising in unstructured P2P overlay networks. One of the factors, packet inter-arrival time variations, could play an important role for prediction of impending congestion on the network. Moreover, the correlation between packet delay and packet loss have been studied extensively by various methods. Various portions of the media blocks ahead of the playing position of audience-peers are fetched (i.e. block 1 of $P_{1,3}$ can be downloaded from $P_{1,1}$ and $P_{1,2}$, but block 2 of $P_{1,3}$ can be downloaded from $P_{1,2}$ only) and temporarily stored in the video packet buffer as shown in Fig. 2 . In the figure, blocks near the playhead positions are considered more significant than others; therefore, the delay trend can be predicted based on the packet size information and timing information (packet inter-arrival times) over the entire session. Other important factors of the audience-peers are the clip names (i.e. clip $x_{1}$ ) and the playing position of the clip. The attributes can support continuous playback and share video segments with nearby peers interested in the same video content, even though peers can change the playing position of the serviced clips at any time with VCR operations (i.e. rewind, forward and pause) on the P2P VoD systems.

A peer has a higher probability of receiving data from closer peers than from far away peers and packet inter-arrival time distribution exhibits hidden properties of the network flow information (i.e. jitter). Let $Z_{t}$ be a discrete-time stochastic process obtained by observing the variance in the arrival rate of packets from peers, while the inter-packet gap is constant at the source peers. $\Delta Z_{t}=Z_{t}-Z_{t-1}$ is the inter-arrival times between the current and the previous packet in an in-order buffer at time $t$. In particular, we extended the original bottleneck detection metric proposed by [23] and a modified kurtosis method is adopted to detect bottleneck on the inter arrival times distribution (depending on the sign of kurtosis) for P2P VoD system. The modified kurtosis for $\mathrm{P} 2 \mathrm{P}$-based service is defined as follows:

$$
\kappa=\sum_{\lambda=1}^{\zeta}\left(\frac{(\zeta-\lambda+1)}{\sum_{v=1}^{\zeta} v} \bullet\left(\frac{1}{n_{\lambda}-1} \sum_{c=1}^{n_{\lambda}}\left(\frac{\Delta Z_{c}-\overline{\Delta Z}}{\sigma}\right)^{4}-3\right)\right)
$$

where $\zeta$ is the cumulative number of peer changed during the periods when there is a movie playing on the window of an audience-peer. Historical kurtosis data of connected peers probably hide near future supportability in the path. Therefore, the first item is a weighting factor, the weighting for each older kurtosis data point decreases by given progressively less weighting. $n_{\lambda}$ is the number of packet inter-arrival times in peer $\lambda, \overline{\Delta Z}$ is the mean of the measured values, and $\sigma$ is the standard deviation. Network bottleneck and detect network abnormities can be identified by a positive kurtosis value. A negative kurtosis value can be seen as a property of underutilized links.

\section{B. Agents Replication and Advertising Strategy}

An agent who patrols the limited broadcasting area (life-time of packets limited by time-to-live (TTL) value) to smooth video playback can place advertising into the online marketing communication channel in order to meet established businesses objectives. The decision whether or not to support data replication for the channel depends both on bottleneck status of peers and dynamic resources of advertising agents. In our proposed method, a density-based clustering method employed as a bottleneck analysis among audience-peers and an algorithm of DENCLUE (DENsity-based CLUstEring) [24] are extended to identify data-support location with some modifications in the 
influence function. All negative kurtosis values can be excluded and changed to zero. Agent $T_{i w}$ organizes its $N$ audience peers into several groups based on the clip names, while the replication process will constantly be computed based on the high bottleneck location for maximizing recovery of high-popular video quality and operational efficiency. Suppose the grouped peers can be represented by a discrete two-dimensional space with coordinates $(k, t)$ where $k$ is the kurtosis-value coordinate and $t$ is the time coordinate (the play position of the clip). The influence function, $f(a, b)$, of peer a to one of its same group peers $b$ is defined as:

$$
f(a, b)=\left\{\begin{array}{cc}
1, & \text { if } \sqrt{\left(a_{k}-b_{\mathrm{k}}\right)^{2}+\left(a_{t}-b_{t}\right)^{2}}>\delta \\
0, & \text { otherwise }
\end{array}\right.
$$

$\delta$ is a density parameter that is used to indicate the time interval between still frames in a movie clip. The density function of peer a, $f^{D}(a)$, results from the influence function of:

$$
f^{D}(a)=\frac{\sum_{i=1}^{N} f(a, b)}{N}
$$

The noise level $\varepsilon_{i w}$ is a cutoff value that indicates whether the aggregated data should be grouped by certain dynamic resources of advertising agents $T_{i w}$. In other words, a lower $\varepsilon_{i w}$ indicates that the resource availability of agent $T_{i w}$ is actually high-score on time $t$. Therefore, the binary representation of grouping process is defined as:

$$
G_{t}^{T_{i w}}(a)= \begin{cases}1, & \text { if } f^{D}(a)>\varepsilon_{i w} \\ 0, & \text { otherwise }\end{cases}
$$

\section{Advertising Plans and Advertisement Publishing Rules}

Advertisement providers $A_{i}$ can create their own advertising plans (i.e. launched date, advertising language, and customized keywords) and advertisement publishing rules can be created based on partial similarity matching of the advertising plans between ads and VoD clips. The publishing rules describe what ads $\left\{f_{i 1}, \ldots, f_{i v}\right\}$ of $A_{i}$ should be associated with clips $\left\{a_{i 1}, \ldots, a_{i u}\right\}$ of $A_{i}$ in P2P VoD system. Agent $T_{i w}$ is based on the movie clip names that are found on the time line of the audience-peers group to pull ads and push them to audience peers in the $\mathrm{P} 2 \mathrm{P}$ online marketing communication channel. Hence, the agents can serve advertisements on target audience-peers.

\section{Simulation Results}

In order to evaluate the validity of the proposed agent-driven advertising framework in a P2P video-on-demand (VoD) based overlay network, we used a CIF version of the standard MPEG test sequences for a movie library and ads videos. Movies in the P2P VoD system expanded in length of clips that follows a normal distribution in range of $10 \mathrm{~min}$ to $20 \mathrm{~min}$, and the length of ads videos are around 40 seconds (15KB 20KB). Those test sequences are encoded using MSRA 3D wavelet video coding software at 15 frames per second and a GOP is composed of 64 frames. The time to live value of broadcasting packet is 7 . Peer reliability of sustaining service follows normal distribution in the range of 0.1 to 0.9 . In addition, the packet inter-arrival time is followed by Weibull distribution [25] and the request distribution of peers is followed by Zipf distribution.

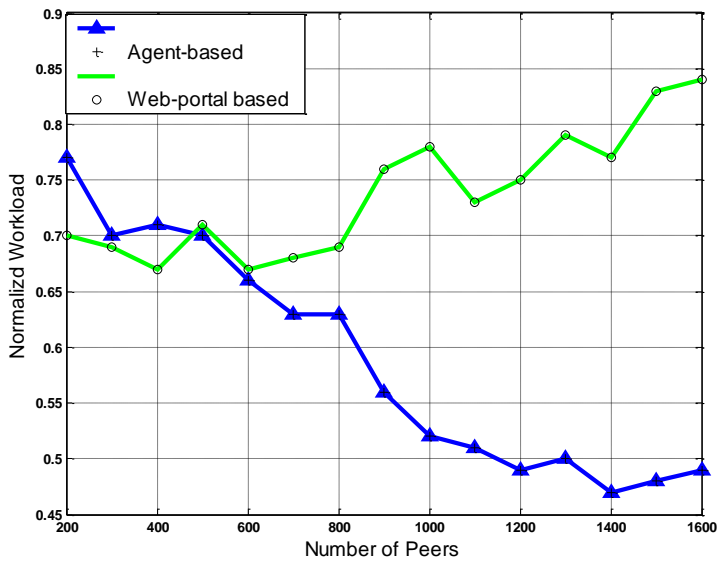

Fig. 3. Workload imposed on the server $(\delta=10$, $\operatorname{ciw}=0.19)$

Fig. 3 shows the workload of VoD server comparison between a web-portal based advertising scheme and agent-driven advertising scheme. The impact of free riding is one of the most commonly discussed problems in $\mathrm{P} 2 \mathrm{P}$ networks. (e.g., nearly $20-40 \%$ of Napster [26] and $85 \%$ of Gnutella [27] users contribute nothing or little resources to cooperative with other peers) may seriously affect system performance. The server load imposed by large numbers of peers, especially free riders, requesting simultaneous can be a performance problem. Thus, the server load imposed under dynamic network conditions with the proportion of peer number is investigated with $40 \%$ of free-riding peers. The measurement reflects the agent-peers scheme and can transform a server load into scalable bandwidths at larger peer-nodes. The agent-peers are sharing ads broadcasting workloads from the web-portal server. In addition, the scheme has significantly increased advertising service quality through improved online marketing communication channels performance.

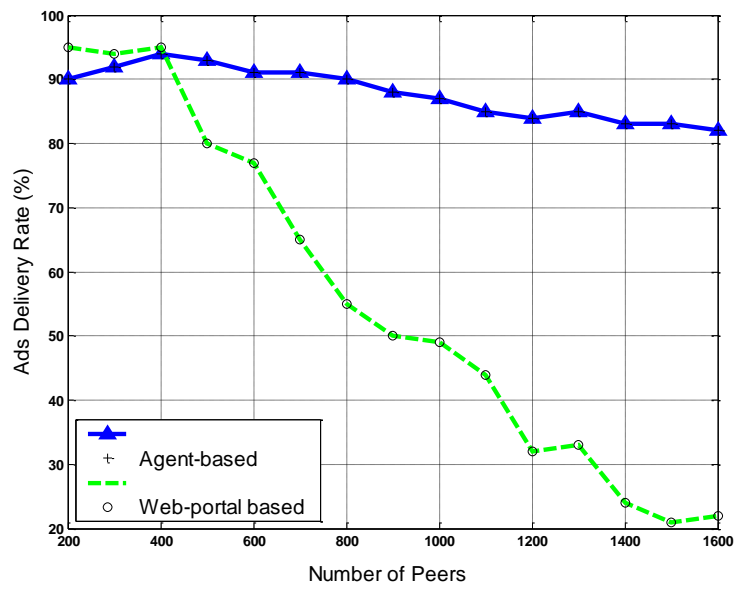

Fig. 4. Delivery rate in different network sizes $(\delta=10$, $\operatorname{ciw}=0.2)$ 
The delivery rate, shown in Fig. 4, varies from 200 to 1600 peers and has simultaneous request access to both the common resource and the network size. The delivery rate is defined as the percentage of audience-peers that successfully received the advertisement at a fixed time period ( $1 \mathrm{~min}$.). All peers communicate in an unstructured way by the network connection speed 1.536 Mbps (DS1/T1). The advertising service is carried by the advertising agents, and the commercial ads can be delivering to the right online audience based on partial similarity matching of the advertising plans between advertisements and the keywords of the clips. The proposed scheme has significantly increased the chance that ads will be promoted on the online marketing communication channel. Each advertising video is inserted into packet buffer of peers from where it is gotten later to play. Agent-driven schemes can achieve significant cooperative gains from obtaining high scalability for larger applications on large $\mathrm{P} 2 \mathrm{P}$ VoD advertising systems.

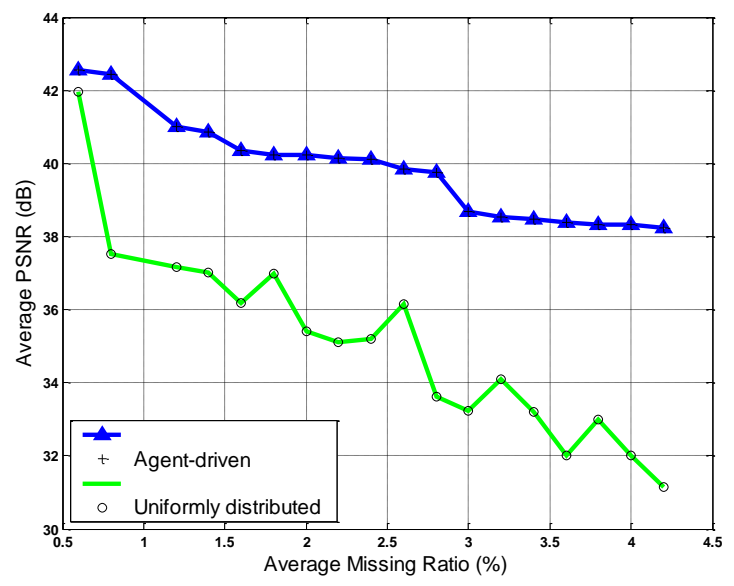

Fig. 5. Impact of VCR operaton in the missing ratio $(\delta=10$, ciw $=0.2)$

To evaluate the impact of VCR operations on video quality, each user performs one VCR request at a random selected point on the clips. Fig. 5 illustrates the average peak signal-to-noise ratio (PSNR) between a uniformly distributed replication scheme and the proposed scheme. The overall response latencies in VCR interactivities are reduced by the higher accuracy proposed scheme which accommodates VCR operations (FORWARD, REWIND, PAUSE and RESUME operations). The issues in the uniformly distributed replication scheme are that all replica locations are treated equally without discrimination, and it is less flexible enough to apply to resource sharing. The result is that the agent-driven scheme is effective to support VCR-like operations and smooth video playback. These findings led to the conclusion that the proposed pre-allocation way is cost effective for multimedia data replication in $\mathrm{P} 2 \mathrm{P}$ VoD system.

\section{CONCLUSION AND FUTURE WORK}

This paper proposes agent-driven framework using peer-level bottleneck detection and agent-level density-based clustering techniques for regional replication strategy in unstructured P2P advertising VoD service. We arrive at the conclusion that a robust online marketing communication channel can provide high video quality and smooth video playback. Also, advertising agents can distribute ads to peers with a highly interactive and responsive current network status of user. Furthermore, a significant conclusion derived from the results of this study is that the robust online marketing communication channel can build up high visibility of advertisement with minimum effort and low delivery time. These findings also demonstrate the scalability of ad serving works impact on the effectiveness of internet advertising's optimal allocations of advertising resources. Moreover, the correlations between the network basis of audience-peer and the strength of ad-messages promoting confirms the practical feasibility of this robust method in $\mathrm{P} 2 \mathrm{P}$ VoD advertising service.

Our future work will be based on the present result of creation to develop a more interactive and immersion-based platform that includes interacting with audience-peers, increasing customer retention, and P2P community-driven advertising. Furthermore, in coordination with the functions of the interactive P2P VoD system, reducing free-riding will assist us in designing incentive mechanisms to contribute to the high-quality and cost-effective P2P online marketing communication channels.

\section{REFERENCES}

[1] J. F. Buford and H. Yu, "Peer-to-Peer Networking and Applications: Synopsis and Research Directions," Handbook of Peer-to-Peer Networking, Springer US, pp. 3-45, 2010.

[2] Zapshares. [Online]. Available: http://www.zapshares.com/

[3] Mediadefender. [Online]. Available: http://www.mediadefender.com/

[4] P2pads. [Online]. Available: http://www.p2pads.com/

[5] Brandassetdigital. [Online]. Available: http://www.brandassetdigital.com

[6] Mininova. [Online]. Available: http://www.mininova.org/

[7] I. J. Taylor, From P2P to Web Services and Grids: Peers in a Client/Server World, Springer, 2004, pp. 102-131.

[8] M. P. Papazoglou, B. J. Kramer, and J. Yang, "Leveraging web-services and peer-to-peer networks," in Proc. the 15th Int. Conf. on Advanced Information Systems Engineering, pp. 15-20, 2003.

[9] Y. Huang, T. Z. Fu, D. Chiu, J. C. Lui, and C. Huang, "Challenges, Design and Analysis of a Large-scale P2P VoD Systems," ACM SIGCOMM, 2008.

[10] Chao Zhang, P. Dhungel, Di Wu, and Keith W. Ross, "Unraveling the BitTorrent Ecosystem," IEEE Transactions on Parallel and Distributed Systems, vol. 22, no. 7, pp.1164-1177, 2011.

[11] C. Li and C. Chen, "Measurement-based study on the relation between users' watching behavior and network sharing in P2P VoD systems," Computer Networks, vol. 54, no. 1, pp. 13-27, 2010.

[12] K. Berte, P. Vyncke, and E. De Bens, "Opportunities of interactive formats for innovative advertising on digital television," European Conference on Interactive Television, pp. 55-58, 2010.

[13] R. E. Bruner and J. Singh, "Video Ad Benchmarks: Average Campaign Performance Metrics," A DoubleClick Research Report, February 2007.

[14] S. Donohue. (2009). Ideal Length for Internet Video Ads: 40 Seconds. [Online]. Available: http://www.contentinople.com/author.asp? section_id=603\&doc_id=177205

[15] R. Owen and P. Humphrey, "The Structure of Online Marketing Communication Channels," Journal of Management and Marketing Research, vol. 2, pp. 54-62, 2009.

[16] Z. Chen, H. Shen, Q. Xu, and X. Zhou, "Instant Advertising in Mobile Peer-to-Peer Networks," IEEE International Conference on Data Engineering, pp. pp. 736-747, 2009.

[17] A. E. Levi and Br. W. Bauer, "Method and System for Peer-to-peer Advertising between Mobile Communication Devices," United States Patent 7664516, February 2010.

[18] N. Salamanos, E. Alexogianni, and M. Vazirgiannis, "Ad-Share: An Advertising Method in P2P Systems based on Reputation Management," 8th Hellenic-European Conference on Computer Mathematics and its Applications (HERCMA), 2007. 
[19] J. Feigenbaum, D. C. Parkes, and D. M. Pennock, "Computational Challenges in E-commerce," Communications of the ACM, vol. 52, no. 1, pp. 70-74, 2009.

[20] Schange. [Online]. Available: http://www.schange.com/

[21] Comcast. [Online]. Available: http://www.comcast.com/

[22] $\mathrm{C}$. $\mathrm{Li}$ and $\mathrm{C}$. Chen, "A neasurement based study on the relations between users' watching behavior and network sharing in $\mathrm{P} 2 \mathrm{P}$ VoD systems," Computer Networks, vol. 54, no. 1, pp. 13-27, 2010.

[23] P. Varga, "Analyzing Packet Interarrival Times Distribution to Detect Network Bottlenecks," in Proc. IFIP WG6.3 Workshop, EUNICE 2005, Balatonfured, Hungary, 2005.

[24] A. Hinneburg and D. A. Keim, "An efficient approach to clustering in large multimedia databases with noise," in Proc. 4th Int. Conf. on Knowledge Discovery and Data Mining, pp. 58-65, 1998.

[25] M. Pustišek, I. Humar, and J. Bešter, "Empirical analysis and modeling of peer-to-peer traffic flows," 14th IEEE Mediterranean Electrotechnical Conference, pp. 169-175, May 2008.

[26] S. Saroiu, P. Krishna Gummadi, and S. D. Gribble, "Measuring and analyzing the characteristics of Napster and Gnutella hosts," Multimedia Systems, vol. 9, no. 2, pp. 170-184, 2003.

[27] D. Hughes, G. Coulson, and J. Walkerdine. (2005). Free riding on Gnutella revisited: the bell tolls. IEEE Distributed Systems Online. [Online]. 6(6). Available: http://ieeexplore.ieee.org/xpls/abs_all.jsp? arnumber $=1471660 \& \operatorname{tag}=1$

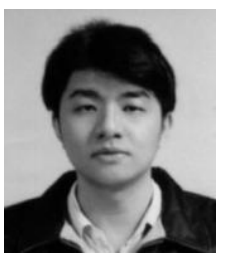

Chien-Peng Ho received his B.S. degree in Computer Science from Tatung University, Taiwan, in 1993, his M.S. degree in Electronic Engineering from National Taiwan University of Science and Technology, in 1995, and his Ph.D. degree in Computer Science from National Chiao Tung University, Taiwan, in 2012

$\mathrm{He}$ is currently a Researcher with Information and Communications Research Laboratories of Industrial Technology Research Institute, Taiwan. His research interests include peer-to-peer networks, multimedia communications, and video coding systems.

Dr. Ho is a senior member of the IACSIT, SIE, and a member of the IEEE, IEICE. He has been involved in the MPEG-21 Testbed project of ISO/IEC TR 21000.

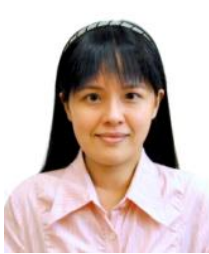

learning.

Prof. Hsieh is the Chief Editor of the journal Teaching Chinese as a Second Language. She is a member of Council in Association of Teaching Chinese as a Second Language. 